\title{
Spatial Distribution of Normal Lung Sounds in Healthy Individuals under Varied Inspiratory Load and Flow Conditions
}

\author{
Manuel Lozano-García, Clare M. Davidson, Carlos Prieto-Ramón, John Moxham, Gerrard F. Rafferty, \\ Caroline J. Jolley and Raimon Jané, Senior Member, IEEE
}

\begin{abstract}
Respiratory sounds yield pertinent information about respiratory function in both health and disease. Normal lung sound intensity is a characteristic that correlates well with airflow and it can therefore be used to quantify the airflow changes and limitations imposed by respiratory diseases. The dual aims of this study are firstly to establish whether previously reported asymmetries in normal lung sound intensity are affected by varying the inspiratory threshold load or the airflow of respiration, and secondly to investigate whether fixed sample entropy can be used as a valid measure of lung sound intensity. Respiratory sounds were acquired from twelve healthy individuals using four contact microphones on the posterior skin surface during an inspiratory threshold loading protocol and a varying airflow protocol. The spatial distribution of the normal lung sounds intensity was examined. During the protocols explored here the normal lung sound intensity in the left and right lungs in healthy populations was found to be similar, with asymmetries of less than $3 \mathrm{~dB}$. This agrees with values reported in other studies. The fixed sample entropy of the respiratory sound signal was also calculated and compared with the gold standard root mean square representation of lung sound intensity showing good agreement.
\end{abstract}

Clinical Relevance - This study provides information on the spatial distribution of normal lung sound intensity in a healthy population during two separate protocols and presents a novel representation of lung sound intensity. The results obtained here in a healthy population may be used for comparison with respiratory sounds recorded in a patient population using similar protocols.

\section{INTRODUCTION}

Respiratory sounds (RS) provide important information about respiratory function in healthy and patient populations, and in the detection, assessment and monitoring of respiratory disease. In current clinical practice, RS are predominantly assessed using a stethoscope. This is subjective and limited by the expertise and hearing ability of the health professional

This work was supported in part by the CERCA Program/Generalitat de Catalunya, in part by the Secretaria d'Universitats i Recerca de la Generalitat de Catalunya under grant GRC 2017 SGR 01770 and in part by the Spanish grant RTI2018-098472-B-I00 (MCIU/AEI/FEDER, UE). C. M. Davidson was the recipient of a BEST postdoctoral fellowship funded by the European Commission under Horizon 2020's Marie-Sklodowska-Curie Actions COFUND scheme (GA 712754) and the Severo Ochoa programme of the Spanish Ministry of Science and Competitiveness (SEV-2014-0425 (20152019)). The funders had no role in study design, data collection and analysis, preparation of the manuscript or the decision to publish.

M. Lozano-García, C. Davidson, C. Prieto-Ramón and R. Jané are with the Institute for Bioengineering of Catalonia (IBEC), The Barcelona Institute involved. Despite the recent advances in sensors and signal processing techniques, the application of RS analysis in clinical practice is not widespread. Therefore, the computational analysis of RS, including the development of a standard analysis methodology, is an important area of research, which holds the potential to be readily translated to a clinical environment. This study aims to extend the knowledge of RS in healthy populations and suggest a new method by which to assess normal lung sound intensity (LSI).

Respiratory sounds are mechanical vibrations caused by turbulent airflow through airways during breathing [1]. In general, inspiratory sounds are approximately $10 \mathrm{~dB}$ louder than expiratory sounds at the chest surface [2]. Accordingly, most studies focus only on the inspiratory sound when analyzing the distribution of the RS amplitude. Significant differences in the spatial distribution of RS have been reported in the literature in patient populations [3], [4], [5]. In a healthy population, an asymmetry of $\pm 3 \mathrm{~dB}$ between the left and right lung sounds has been shown to be a normal range, with the LSI of the left side generally higher than that of the right [6], [7]. The reasons for this asymmetry are not fully understood but are thought to be related to anatomical differences and differences in regional flow turbulence and sound generation [8]. Dominance on the left side during only the louder inspiratory phase has also been suggested [9].

Knowledge of normal LSI at different locations in the lungs can provide useful information about local changes in airflow limitation that occur in respiratory disease [4]. LSI is generally calculated using the root mean square (RMS) method which tracks the envelope of the sound signal. However, this quantity is affected by any impulsive noise contaminating the signal. Sources of impulsive noise in RS signals include cardiac activity and microphone clicks as well as other external sounds. Sample entropy [10] is a measure of complexity of a signal with a lower entropy associated with a signal with a higher regularity. Sample entropy is calculated at once for the entire signal, whereas fixed sample entropy

of Science and Technology (BIST), UPC Campus Diagonal-Besòs, Av d'Eduard Maristany, 10-14, building C, 5th floor, 08930, Barcelona, Spain (e-mails: mlozano, cdavidson, cprieto, rjane \{@ibecbarcelona.eu\}). M Lozano-García and R. Jané are also with CIBER-BBN and Universitat Politècnica de Catalunya (UPC)-Barcelona Tech.

J. Moxham, G. F. Rafferty, and C. J. Jolley are with the Centre of Human \& Aerospace Physiological Sciences, School of Basic \& Medical Biosciences, Faculty of Life Sciences \& Medicine, King's College London, King's Health Partners, London, United Kingdom (e-mails: john.moxham, gerrard.rafferty, caroline.jolley $\{@$ kcl.ac.uk\}). G. F. Rafferty and C. J. Jolley are also with King's College Hospital NHS Foundation Trust, King's Health Partners, London, United Kingdom. 
(fSampEn) is calculated within a moving window using fixed parameters for each window. Fixed sample entropy has been shown to be less sensitive than the RMS to the effects of cardiac activity in electromyographical signals [11], [12].

This study examines the effect of varying the inspiratory threshold load and the airflow of respiration in healthy individuals on the spatial distribution of normal LSI. The protocols used permit the exploration of a range of variations in breathing such as those physiologically employed in response to external stressors. In addition, the novel representation of the LSI by the fSampEn of the RS signal was explored and compared with the current gold standard RMS estimator of LSI.

\section{METHODS}

\section{A. Study Population}

Respiratory sound and airflow were recorded from twelve healthy subjects ( 6 female), age 33 (30-38) years, BMI 22.2 $(20.6-24.2) \mathrm{kg} / \mathrm{m}^{2}, \mathrm{FEV}_{1} 98.0(94.8-105.5) \%$ of predicted and FVC 105.0 (91.5-110.2) \% of predicted. All values are median with the interquartile range shown in parentheses. Subjects had no history of cardiorespiratory disease and were familiar with physiological studies. The study was conducted in accordance with the Declaration of Helsinki with approval obtained from the NHS Health Research Authority (NRES Committee London - Dulwich 05/Q0703). Fully informed written consent was given by all participants prior to inclusion. All data were acquired at the King's College Hospital, London, United Kingdom.

\section{B. Experimental Protocol}

The experimental protocol consisted of two parts: an inspiratory threshold loading protocol and a varying flow protocol. The inspiratory threshold loading protocol simulates different levels of effort while breathing, whereas varying the airflow relates to changes in the speed and volume of breathing. Each subject was seated, with a noseclip in place, and breathed through a pneumotachograph (4830; Hans Rudolph Inc., KS, USA) via a mouthpiece throughout.

An initial maximal static inspiratory pressure (PImax) maneuver was performed and repeated several times to ensure maximal volitional effort. The inspiratory threshold load levels for the subject were calculated as $0 \%$ (L0), $12 \%$ (L1), $24 \%$ (L2), 36\% (L3), 48\% (L4) and 60\% (L5) of the PImax. The subject then performed 30 breath cycles at each prescribed level in ascending order, separated by rest periods in which all respiratory measurements returned to baseline. The inspiratory threshold loads were generated with an electronic inspiratory muscle trainer (POWERbreathe K5; POWERbreathe International Ltd, Southam, UK) which was attached to the distal end of the pneumotachograph. This loading protocol is outlined in detail in [12].

For the varying flow protocol, a maximal inspiratory flow (MIF) maneuver was performed and repeated several times, again, to ensure maximal volitional effort. Four target levels of airflow were designed based on MIF with level 1 corresponding to normal quiet breathing and 3 intermediary levels defined in between this and the MIF of the subject. During the data acquisition, the subject could see their airflow signal on a computer screen together with a guideline

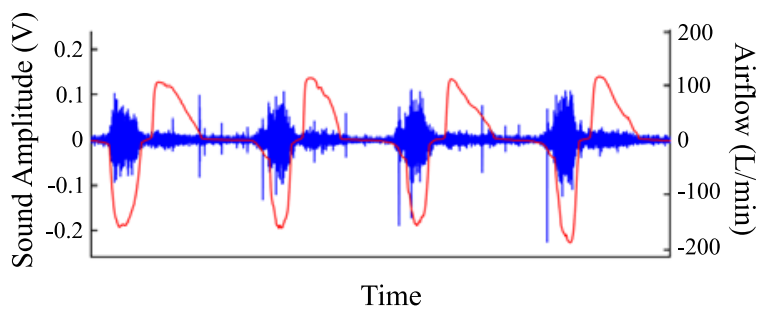

Figure 1: Example of airflow and a single channel of lung sound recorded during the prescribed protocol. The airflow is shown in red and the lung sound is plotted in blue. The respiratory sound is contaminated by impulsive noise.

indicating the target flow level during each inspiration. The subject then performed several breath cycles at each of the four defined levels in ascending order, separated by rest periods in which all respiratory measurements returned to baseline.

\section{Data Acquisition}

Respiratory sounds were recorded using four piezoelectric contact microphones (TSD108, Biopac Systems, Inc.) placed on the posterior skin surface of the subject. The microphones were positioned bilaterally at the base and near the upper lobes of the lungs and attached with double-sided adhesive discs. Airflow was acquired with a pneumotachograph (4830; Hans Rudolph Inc., KS, USA) connected to a differential pressure transducer (DP45; Validyne Engineering, CA, USA) and amplifier (CD72; Validyne Engineering, CA, USA). Respiratory sound and airflow signals were sampled at $10 \mathrm{kHz}$ and $100 \mathrm{~Hz}$, respectively, using a 16-bit analogue-to-digital converter (PowerLab 16/35; ADInstruments Ltd, Oxford, UK). An example of the acquired raw signals is shown in Fig. 1 , where respiratory sounds are contaminated by impulsive noise.

\section{Data Analysis}

Respiratory sound data were resampled at $4000 \mathrm{~Hz}$, and bandpass filtered between 70 and $2000 \mathrm{~Hz}$ with an 8th order, zero-phase Butterworth filter. A $50 \mathrm{~Hz}$ comb filter was applied to remove mains interference and associated harmonics.

A zero-crossing detector was applied to the flow signal in order to identify the respiratory phases of each cycle. As inspiratory sounds are more clearly heard over the chest surface than expiratory sounds [2], only the inspiratory cycles were analyzed here. For each segmented inspiratory phase, the difference from the median value of the inspiratory time, volume of inspiration and peak flow of inspiration were calculated. These values were summed and used to rank the inspiratory phases, with the first cycle lying closest to the median value. The RS signals of the inspiratory cycles were then examined iteratively, and a cycle accepted for further processing if it contained no outliers (defined as $>3$ std. dev. from the mean) in any of its 4 channels. Ten cycles were selected for each level of the inspiratory threshold load protocol and the varying flow protocol, resulting in 100 cycles for each subject.

The LSI was estimated in two separate ways: by calculating the RMS amplitude and by calculating the fSampEn of the signal. The fSampEn was calculated over a moving window using a fixed tolerance $r=0.5 *$ ref, and an embedding dimension $\mathrm{m}=2$. The ref is defined for each subject and protocol as the mean standard deviation of the 

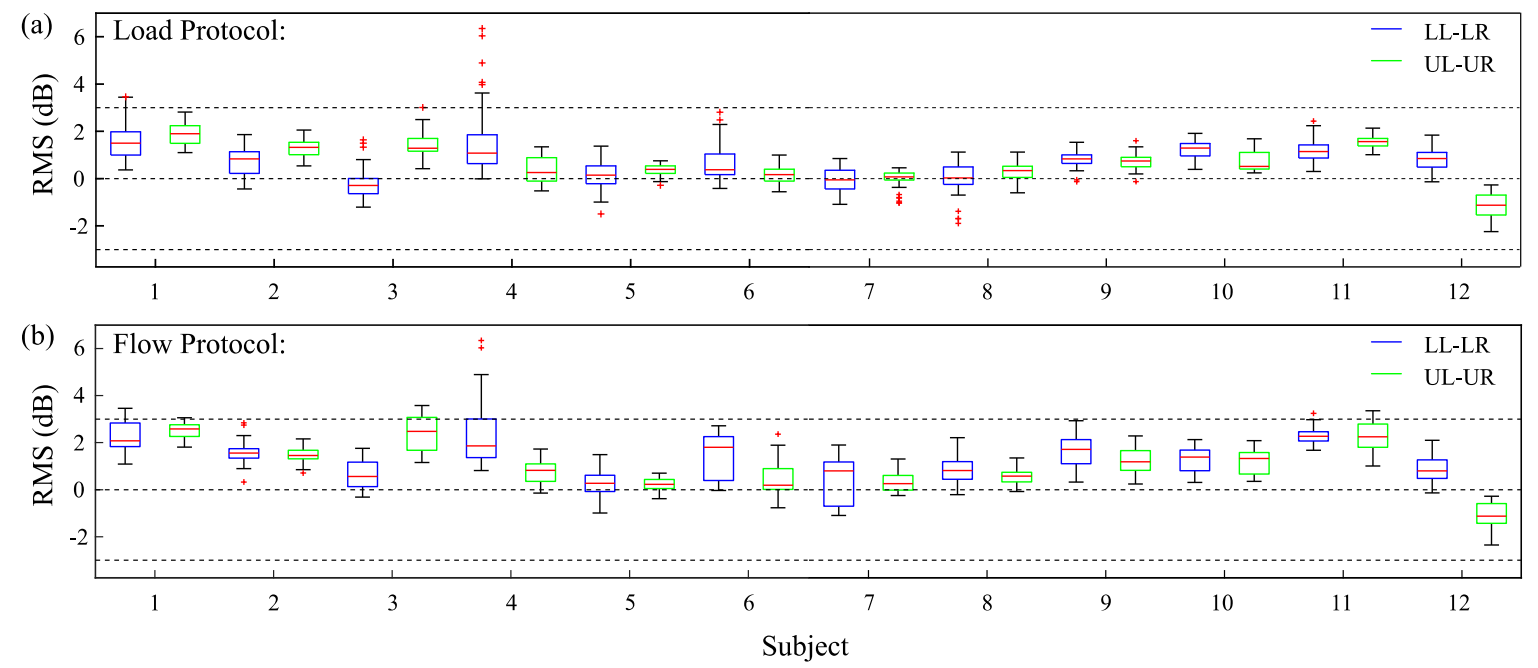

Figure 2. Differences in spatial distribution of normal lung sound intensity (LSI) recorded in 12 healthy subjects during (a) an incremental inspiratory threshold loading protocol and (b) a varying flow protocol, calculated using the root mean square (RMS). Each subject is represented by two columns, the first corresponding to the ratio between the lower left and right lungs (blue), and the second corresponding to the ratio between the upper left and right lungs (green). The $\pm 3 \mathrm{~dB}$ thresholds and $0 \mathrm{~dB}$ are indicated with black dashed lines.

segmented cycles from all four RS channels for all load or flow levels.

Data analysis was performed offline using custom developed scripts (MATLAB 9.6, The MathWorks Inc., Natick, MA, USA, 2019).

\section{RESULTS}

The median difference in normal LSI between the upper and lower, left and right lungs was below $\pm 3 \mathrm{~dB}$ during both the inspiratory threshold loading protocol and the varying flow protocol for all twelve subjects (Fig. 2). For each subject, the median is based on 60 measurements for the inspiratory loading threshold protocol and 40 measurements for the varying flow protocol. Two subjects had a median left to right ratio of $<0 \mathrm{~dB}$ for the upper portion of the lungs and one subject for the lower portion. For the remaining nine subjects, all other median left to right ratios were positive, indicating that in general, the LSI in the left lung is slightly higher than in the right. The LSI for Fig. 2 was calculated using the RMS.

Root mean square and fSampEn were both used to obtain estimates of normal LSI. The fSampEn of the signal was not influenced by impulsive noise which occurred in some of the RS cycles whereas the RMS, yielding the envelope of the signal, tracked these spikes (Fig. 3). Several fSampEn parameters were tested in the calculation of LSI with the chosen parameters distinguishing RS most clearly from background noise.

The spatial distribution of normal LSI compared between the left and right, lower and upper lungs calculated using fSampEn was observed to be consistent with that calculated using the RMS method (Fig. 4 and Fig. 5). Data from both the inspiratory loading threshold protocol (red circles) and the varying flow protocol (black circles) for a single subject are included in Fig. 5, with each data point representing the mean value calculated over 10 breath cycles for a given load or flow.

\section{DISCUSSION}

The main finding of this study is that the ratio of left to right, upper and lower, median values of normal LSI in a healthy population during an inspiratory threshold loading protocol and a varying flow protocol have a magnitude less than $3 \mathrm{~dB}$. Although within the normal range, the results showed higher LSI in the left rather than right side for both the upper and lower lungs, and for most subjects. Similar values have previously been shown in healthy individuals [6], [13]. However, an inspiratory threshold loading protocol has not previously been applied to quantify RS, as it is a protocol normally employed to evaluate muscular function. The addition of inspiratory threshold loads during respiration simulates different levels of effort while breathing.

As previous studies have reported, airflow has a direct relationship with LSI [14], [15]. In this study, the airflow and the LSI were observed to increase as the imposed load increased. It is interesting to note though, that individual subjects adapted to the applied loads in various ways, with some increasing their flow rate in a close to linear manner as the load increased, and others increasing their flow rate in a more logarithmic way.

An additional result of this study was the demonstration of fSampEn as a robust estimator of LSI. Fixed sample entropy was originally proposed in [10] as a statistical method to analyze data from nonlinear dynamic systems and is a method well suited to the analysis of physiological data. The fSampEn method compares favorably with the gold standard RMS

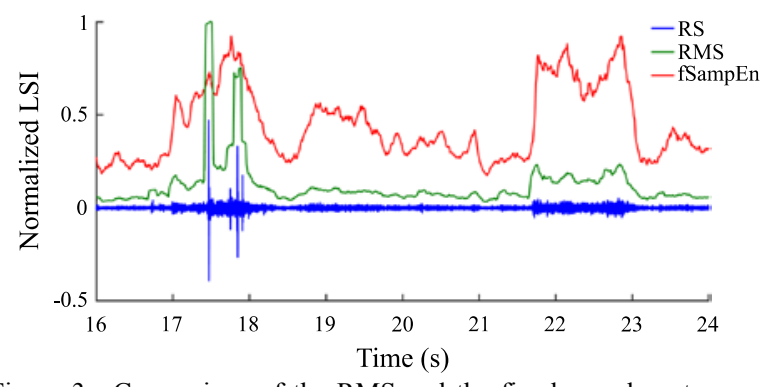

Figure 3. Comparison of the RMS and the fixed sample entropy (fSampEn) as estimators of the lung sound intensity (LSI) in respiratory sound with and without impulsive noise. The RMS is shown in green and the fSampEn is shown in red, with the original sound signal plotted in blue. 

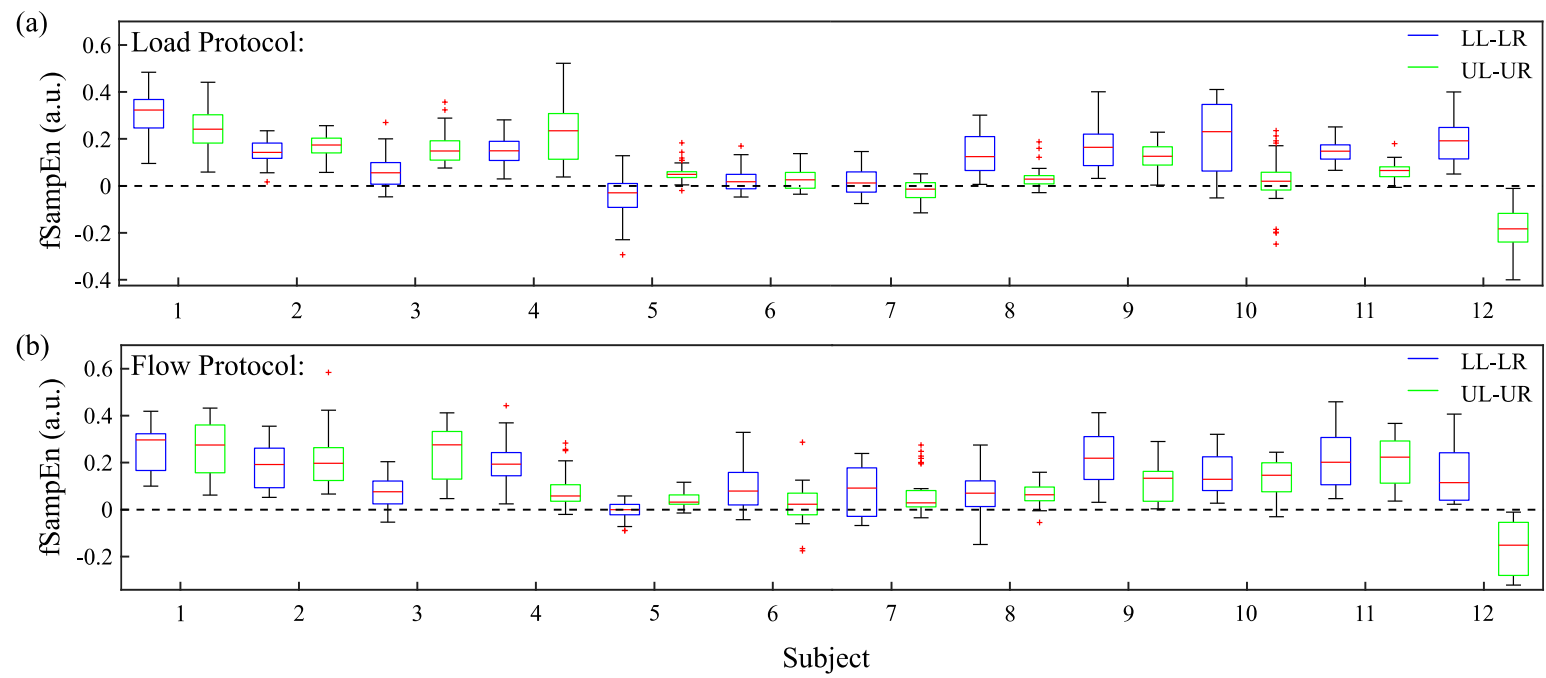

Figure 4. Differences in spatial distribution of normal lung sound intensity (LSI) recorded in 12 healthy subjects during (a) an incremental inspiratory threshold loading protocol and (b) a varying flow protocol and calculated using fixed sample entropy (fSampEn). Each subject is represented by two columns, the first corresponding to the ratio between the lower left and right lungs (blue), and the second corresponding to the ratio between the upper left and right lungs (green).

method, with the main advantage being that the fSampEn is not affected by impulsive noise in the signal. A similar spatial distribution of RS was found using both methods. The comparison of normal LSI estimated using fSampEn with those estimated using the RMS method focused on the shape of the curve as the units are different. Entropy is calculated in arbitrary units which does not allow direct comparison with other measurements in terms of absolute values and makes the true physical meaning difficult to interpret.

This study has shown that varying the inspiratory threshold load and airflow during respiration does not affect the spatial distribution of RS in a healthy population. This result can be used as a basis upon which to assess the response of patient populations to similar protocols. In addition, it contributes to the current knowledge of RS in healthy populations. The favorable comparison of using fSampEn to estimate LSI with the RMS method suggests that this is a robust method that could be employed in future studies. It would be particularly useful in cases of high activity or high flow levels where the data contains impulsive noise.
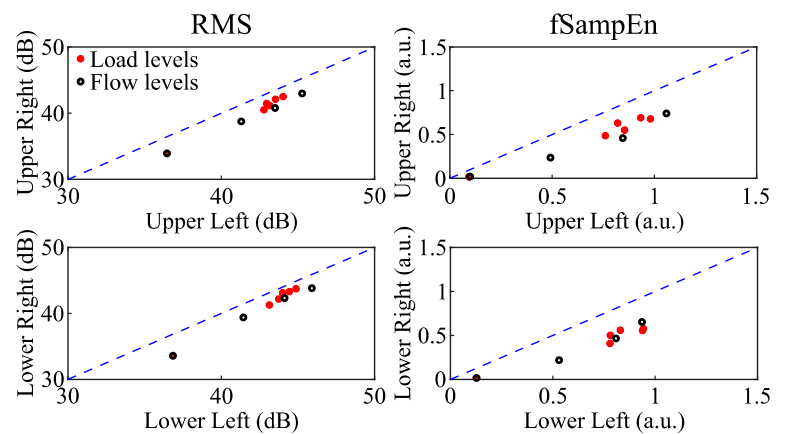

Figure 5. Spatial distribution of lung sound intensity (LSI) recorded in a healthy individual (subject 1 ) during both an incremental inspiratory threshold loading protocol (red circles) and a varying flow protocol (black circles). The relationship between the upper left and right lungs and the lower left and right lungs are both illustrated. The distribution is shown for LSI values calculated using the RMS on the left and using fixed sample entropy (fSampEn) on the right. The blue dashed line indicates a 1:1 ratio between the left and right sides.

\section{REFERENCES}

[1] A. Bohadana, G. Izbicki, and S. S. Kraman, "Fundamentals of lung auscultation," New England Journal of Medicine. 2014.

[2] M. Kompis, H. Pasterkamp, Y. Oh, and G. R. Wodicka, "Distribution of inspiratory and expiratory respiratory sound intensity on the surface of the human thorax," Annu. Int. Conf. IEEE Eng. Med. Biol. - Proc., 1997.

[3] R. Mor, I. Kushnir, J. J. Meyer, J. Ekstein, and I. Ben-Dov, "Breath sound distribution images of patients with pneumonia and pleural effusion," Respir. Care, 2007.

[4] J. A. Fiz, R. Jané, M. Lozano, R. Gómez, and J. Ruiz, "Detecting unilateral phrenic paralysis by acoustic respiratory analysis," PLoS One, 2014.

[5] R. González-Camarena, S. Charleston-Villalobos, A. AngelesOlguín, and T. Aljama-Corrales, "Imaging the thoracic distribution of normal breath sounds," Methods Inf. Med., 2010.

[6] H. Pasterkamp, S. Patel, and G. R. Wodicka, "Asymmetry of respiratory sounds and thoracic transmission," Med. Biol. Eng. Comput., 1997.

[7] A. Oliveira and A. Marques, "Respiratory sounds in healthy people: A systematic review," Respiratory Medicine. 2014.

[8] H. Pasterkamp, S. S. Kraman, and G. R. Wodicka, "State of the Art Respiratory Sounds Advances Beyond the Stethoscope," Am J Respir Crit Care Med, vol. 156, pp. 974-987, 1997.

[9] R. P. Dellinger, J. E. Parrillo, A. Kushnir, M. Rossi, and I. Kushnir, "Dynamic visualization of lung sounds with a vibration response device: A case series," Respiration, 2008.

[10] J. S. Richman and J. R. Moorman, "Physiological time-series analysis using approximate and sample entropy," Am. J. Physiol. Hear. Circ. Physiol., 2000.

[11] L. Estrada, A. Torres, L. Sarlabous, and R. Jané, "Improvement in Neural Respiratory Drive Estimation From Diaphragm Electromyographic Signals Using Fixed Sample Entropy," IEEE J. Biomed. Heal. Informatics, 2016.

[12] M. Lozano-García et al., "Surface mechanomyography and electromyography provide non-invasive indices of inspiratory muscle force and activation in healthy subjects," Sci. Rep., vol. 8, no. 1, pp. 1-13, 2018.

[13] N. Gavriely and D. W. Cugell, "Airflow effects on amplitude and spectral content of normal breath sounds.," J. Appl. Physiol., 1996.

[14] S. S. Kraman, "The relationship between airflow and lung sound amplitude in normal subjects," Chest, 1984.

[15] A. Yadollahi and Z. M. K. Moussavi, "A robust method for estimating respiratory flow using tracheal sounds entropy," IEEE Trans. Biomed. Eng., 2006. 